\title{
MIMO Detection Schemes with Interference and Noise Estimation Enhancement
}

\author{
Pei Xiao, Member, IEEE, Jinsong Wu, Member, IEEE, Colin F. N. Cowan, Senior Member, IEEE
}

\begin{abstract}
Different detection schemes for multiple-input, multiple-output (MIMO) systems are investigated. By enhancing the interference and noise estimation, we propose a novel MIMO receiver strategy, which is shown to achieve superior performance with moderate increase in computational complexity compared to conventional MIMO detection schemes.
\end{abstract}

\section{INTRODUCTION}

Communication systems employing multiple antennas at both the transmitter and the receiver have recently received increased attention due to their ability to provide great capacity increases in a wireless fading environment [1], [2]. However, MIMO systems require high signal to noise plus interference ratio to mitigate the co-channel interference (CCI) and to achieve their potential capacity. In spatial division multiplexing (SDM) systems [2], [3], the transmitted data sequence is transformed into parallel sequences and each sequence is transmitted from a different antenna at the same time with the same carrier frequency. Therefore, the total transmission rate increases in proportion to the number of transmit antennas. At the receiver, it is necessary to separate the signals transmitted from different antennas. For such SDM systems, it is well known that the optimal maximum likelihood detector (MLD) has a prohibitively high complexity, which makes it inapplicable for practical systems. Fixed complexity sphere decoding techniques have been introduced in [4], [5] to approach the performance of the MLD with much reduced complexity. A constraint-depth maximum a posteriori (MAP) MIMO detector based on a trellis representation of the MIMO signals is proposed in [6]. However, these near-optimal detectors still have a high computational complexity which does not facilitate their practical implementation. Among different SDM techniques, vertical Bell Labs Layered space-time (V-BLAST) [3] exhibits a good tradeoff between performance and complexity. In the V-BLAST architecture, a successive interference cancellation (SIC) and nulling algorithm is used to detect the transmitted symbols, such a decision feedback detection mechanism is combined with a channel dependent detection ordering process [3]. Some efficient and fast implementations of the V-BLAST algorithm have been introduced in [7], [8]. In order to approach the near-optimal performance,

Dr. Pei Xiao and Prof. Colin Cowan is with the Institute of ECIT, Queen's University Belfast, BT3 9DT, UK and Dr. Jinsong $\mathrm{Wu}$ is with the department of Electrical and Computer Engineering, Queen's University, Kingston, Ontario, K7L 3N6, Canada. E-mail: \{pei.xiao, c.cowan\}@ecit.qub.ac.uk, wujs@ieee.org this SIC based detection scheme is complemented by maximum likelihood detection in [9] as well as lattice and list reduction scheme in [10]. An improved VBLAST scheme using soft-input, soft-output, and soft-feedback is presented in [11], where the authors propose to make the symbol decision by minimizing the power of the interference plus noise, given a priori probabilities of undetected layer symbols and a posteriori probabilities of past detected layer symbols.

In addition to the V-BLAST based detection schemes, the iterative receiver structure based on the turbo processing principle [12] is a promising alternative. It has been shown in [13], [14] that iterative detection provides an effective means to combat $\mathrm{CCI}$ and to approach the capacity offered by MIMO systems. The detector first forms a soft replica of each composite interfering signal using the log-likelihood ratio (LLR), and subtracts it from the received signal vector. Adaptive filtering then takes place to suppress the interference residual. The filter taps are adjusted according to the minimum-mean-square-error (MMSE) criterion, and the LLR is calculated for the adaptive filter output. This process is repeated in an iterative fashion to enhance the detection performance [14] [16]. To combat frequency-selective fading in high datarate applications, this kind of iterative scheme has been extended to MIMO-OFDM systems [17] and MIMO single-carrier systems with cyclic prefix and frequency domain equalization [18].

In this paper, we show that the existing interference cancellation and nulling based receiver designs are suboptimum and their performance can be improved by exploitation of the rotational variance of the interference. We shall use the following notations: $(\cdot)^{\mathcal{T}}$ denotes matrix transpose, $(\cdot)^{\mathcal{H}}$ matrix conjugate transpose, $(\cdot)^{*}$ matrix conjugate, $\mathrm{E}[\cdot]$ expectation, and $\mathbf{I}_{N}$ an $N \times N$ identity matrix.

\section{SYSTEM MODEL}

We consider a MIMO system with $N_{t}$ transmit antennas and $N_{r}$ receive antennas. The transmitted symbol vector at the $u$ th transmission interval $\mathbf{x}(u)=$ $\left[\begin{array}{lll}x_{1}(u) & \ldots & x_{N_{t}}(u)\end{array}\right]^{\mathcal{T}}$ comprises the transmit symbol (belonging to a finite alphabet $\mathcal{X}$ ) of $N_{t}$ parallel data streams. The data symbols are assumed to be uncorrelated and to have zero mean and unit energy, i.e., $\mathrm{E}\left[\mathbf{x}(u)(\mathbf{x}(u))^{\mathcal{H}}\right]=\mathbf{I}_{N_{t}}$. Using the standard linear channel model, the received signal can be expressed in a vector 
form as

$$
\begin{aligned}
\mathbf{r}(u) & =\mathbf{S}(u) \mathbf{x}(u)+\mathbf{n}(u) \\
& =\sum_{k=1}^{N_{t}} \mathbf{s}_{k}(u) x_{k}(u)+\mathbf{n}(u) \in \mathbb{C}^{N_{r} \times 1},
\end{aligned}
$$

where $\mathbf{r}(u)=\left[\begin{array}{llll}r_{1}(u) & \ldots & r_{N_{r}}(u)\end{array}\right]^{\mathcal{T}}$ is the received signal vector; $\mathbf{n}(u)=\left[\begin{array}{lll}n_{1}(u) & \ldots & n_{N_{r}}(u)\end{array}\right]^{\mathcal{T}}$ denotes the complex additive white Gaussian noise vector with zero mean and covariance matrix $\sigma_{n}^{2} \mathbf{I}_{N_{r}}$, i.e., $\mathbf{n}(u) \sim$ $\mathcal{C N}\left(\mathbf{0}, \sigma_{n}^{2} \mathbf{I}_{N_{r}}\right)$. The channel matrix $\mathbf{S}(u) \in \mathbb{C}^{N_{r} \times N_{t}}$ contains the complex channel gains between every transmit and receive antenna pair at the $u$ th transmission interval, and $\mathbf{s}_{i}(u)$ is the $i$ th column of $\mathbf{S}(u)$. The index $u$ will be omitted in the rest of the paper to simplify the notations whenever no ambiguity arises.

\section{ItERATIVE MIMO DETECTION SCHEMES}

In this section, we first introduce the conventional iterated soft decision interference cancellation (ISDIC) scheme and its modified version, then propose a novel MIMO detection strategy in Section III-C.

\section{A. Conventional ISDIC scheme}

To decode the symbol transmitted from the $k$ th transmit antenna $x_{k}$, replicas of the interference from different transmit antennas can be generated and subtracted from the received signal, i.e.,

$$
\begin{aligned}
\mathbf{r}_{k}^{m} & =\mathbf{r}-\mathbf{S d}_{k}^{m}=\mathbf{S}\left[\mathbf{x}-\mathbf{d}_{k}^{m}\right]+\mathbf{n} \in \mathbb{C}^{N_{r} \times 1} ; \\
k & =1, \ldots, N_{t},
\end{aligned}
$$

where $\mathbf{r}_{k}^{m}$ is the interference canceled version of $\mathbf{r} ; m$ is the iteration index, and the vector

$$
\mathbf{d}_{k}^{m}=\left[\begin{array}{lllllll}
d_{1}^{m-1} & \ldots & d_{k-1}^{m-1} & 0 & d_{k+1}^{m-1} & \ldots & d_{N_{t}}^{m-1}
\end{array}\right]^{\mathcal{T}}
$$

contains the soft estimate of the interference symbols from the previous iteration. This process can be viewed as transforming a MIMO channel into a single-input multiple-output (SIMO) channel [19]. Provided that the CCI is perfectly canceled, it is equivalent to $N_{r}$ antenna diversity reception with single antenna transmission.

In order to further suppress the residual interference in $\mathbf{r}_{k}^{m}$, an instantaneous linear filter is applied to $\mathbf{r}_{k}^{m}$, to obtain $z_{k}=\left(\mathbf{h}_{k}^{m}\right)^{\mathcal{H}} \mathbf{r}_{k}^{m}$, where the filter coefficient vector $\mathbf{h}_{k}^{m} \in \mathbb{C}^{N_{r} \times 1}$ is chosen by minimizing $e_{k}=$ $\mathrm{E}\left\{\left|\left(\mathbf{h}_{k}^{m}\right)^{\mathcal{H}} \mathbf{r}_{k}^{m}-x_{k}\right|^{2}\right\}$, and is derived as $\mathbf{h}_{k}^{m}=\left[\mathbf{S} \mathbf{E}_{k}^{m} \mathbf{S}^{\mathcal{H}}+\right.$ $\left.\sigma_{n}^{2} \mathbf{I}\right]^{-1} \mathbf{s}_{k}$. The matrix $\mathbf{E}_{k}^{m} \in \mathbb{R}^{N_{t} \times 1}$ is formed as

$$
\begin{aligned}
& \mathbf{E}_{k}^{m}=\operatorname{diag}\left\{\operatorname{var}\left(x_{1}^{m-1}\right) \quad \ldots \quad \operatorname{var}\left(x_{k-1}^{m-1}\right) \quad 1\right. \\
& \left.\operatorname{var}\left(x_{k+1}^{m-1}\right) \quad \ldots \quad \operatorname{var}\left(x_{N_{t}}^{m-1}\right)\right\},
\end{aligned}
$$

where $\operatorname{var}\left(x_{j}^{m-1}\right)=\mathrm{E}\left[\left|x_{j}-d_{j}^{m-1}\right|^{2}\right]$. Passing $\mathbf{r}_{k}^{m}$ through the filter $\mathbf{h}_{k}^{m}$ yields the filter output for the $k$ th symbol as $z_{k}^{m}=\left(\mathbf{h}_{k}^{m}\right)^{\mathcal{H}} \mathbf{r}_{k}^{m}=\mu_{k}^{m} x_{k}+i_{k}^{m}$, where $\mu_{k}^{m}=\left(\mathbf{h}_{k}^{m}\right)^{\mathcal{H}} \mathbf{s}_{k}$, and $i_{k}^{m} \sim \mathcal{C N}\left\{0, \sigma_{x}^{2} \mu_{k}^{m}\left(1-\mu_{k}^{m}\right)\right\}$ denotes the combined interference residual and noise. Refer to [14]-[16] for a detailed description of this conventional algorithm.

\section{B. Modified ISDIC scheme}

The performance of the conventional ISDIC scheme can be improved if we not only process $\mathbf{r}_{k}^{m}$, but also its conjugated version $\left(\mathbf{r}_{k}^{m}\right)^{*}$ in order to derive the filter output, i.e., $f_{k}^{m}=\mathbf{a}_{k}^{m} \mathbf{r}_{k}^{m}+\mathbf{b}_{k}^{m}\left(\mathbf{r}_{k}^{m}\right)^{*}=\boldsymbol{\alpha}_{k}^{\mathcal{H}} \mathbf{y}_{k}^{m}$, where $\boldsymbol{\alpha}_{k}^{m}=$ $\left[\begin{array}{ll}\mathbf{a}_{k}^{m} & \mathbf{b}_{k}^{m}\end{array}\right]^{\mathcal{H}}$ and $\mathbf{y}_{k}^{m}=\left[\begin{array}{ll}\left(\mathbf{r}_{k}^{m}\right)^{\mathcal{T}} & \left(\left(\mathbf{r}_{k}^{m}\right)^{*}\right)^{\mathcal{T}}\end{array}\right]^{\mathcal{T}}$. This is the idea of widely linear processing (WLP) [20] which has found many applications in communications systems. For example, an MMSE equalizer and a decision-feedback equalizer (DFE) employing WLP and implemented via finite impulse response (FIR) filters for a MIMO frequency selective channel have been proposed in [21] and [22], respectively. It was concluded that the use of WLP yields considerable performance improvements at the cost of only a limited increase in complexity compared to conventional linear processing. However, the application of the WLP in the literature, including those mentioned above, is mostly confined to the real-valued signal constellations. The application of WLP to complex signals has been addressed in several papers, e.g., in [23], [24] where WLP was applied to complex signals which become rotationally variant due to the use of space-time block coding or widely linear space-time mapping. It was shown in [25] that significant gains can be obtained for complex signals if the rotational variance of the residual interference arising in the course of the iterations of an ISDIC is regarded. Based on this finding, a modified ISDIC scheme was proposed in [25] for iterative multiuser detection in DS-CDMA systems. Here, we extend this algorithm to the MIMO system under study.

Following the modified ISDIC scheme [25], the filter $\boldsymbol{\alpha}_{k}^{m}$ can be derived by minimizing the MSE E $\left\{\left|e_{k}^{m}\right|^{2}\right\}$, where $e_{k}^{m}=f_{k}^{m}-x_{k}$ and $f_{k}^{m}=\left(\boldsymbol{\alpha}_{k}^{m}\right)^{\mathcal{H}} \mathbf{y}_{k}^{m}$ is the filter output. According to the orthogonality principle, the error of the optimal estimator (in a mean square error sense) is orthogonal to the observation [26], i.e., $\mathrm{E}\left[\mathbf{y}_{k}^{m}\left(e_{k}^{m}\right)^{*}\right]=$ $\mathrm{E}\left[\mathbf{y}_{k}^{m}\left(\left(\boldsymbol{\alpha}_{k}^{m}\right)^{\mathcal{H}} \mathbf{y}_{k}^{m}-x_{k}\right)^{\mathcal{H}}\right]=\mathbf{0}$, leading to the solution

$$
\boldsymbol{\alpha}_{k}^{m}=\left(\mathrm{E}\left[\mathbf{y}_{k}^{m}\left(\mathbf{y}_{k}^{m}\right)^{\mathcal{H}}\right]\right)^{-1} \mathrm{E}\left[\mathbf{y}_{k}^{m} x_{k}^{*}\right]=\mathbf{\Psi}_{\mathbf{y y}}^{-1} \Psi_{\mathbf{y} x},
$$

where

$$
\begin{aligned}
& \mathbf{\Psi}_{\mathbf{y y}}=\mathrm{E}\left\{\mathbf{y}_{k}^{m}\left(\mathbf{y}_{k}^{m}\right)^{\mathcal{H}}\right\}=\mathrm{E}\left\{\left[\begin{array}{c}
\mathbf{r}_{k}^{m} \\
\left(\mathbf{r}_{k}^{m}\right)^{*}
\end{array}\right]\left[\begin{array}{ll}
\left(\mathbf{r}_{k}^{m}\right)^{\mathcal{H}} & \left(\mathbf{r}_{k}^{m}\right)^{\mathcal{T}}
\end{array}\right]\right\} \\
& =\left[\begin{array}{cc}
\mathbf{S E}_{k}^{m} \mathbf{S}^{\mathcal{H}}+\sigma_{n}^{2} \mathbf{I} & \mathbf{S} \tilde{\mathbf{E}}_{k}^{m} \mathbf{S}^{\mathcal{T}} \\
\mathbf{S}^{*}\left(\tilde{\mathbf{E}}_{k}^{m}\right)^{*} \mathbf{S}^{\mathcal{H}} & \mathbf{S}^{*} \mathbf{E}_{k}^{m} \mathbf{S}^{\mathcal{T}}+\sigma_{n}^{2} \mathbf{I}
\end{array}\right] ; \\
& \boldsymbol{\Psi}_{\mathbf{y} x}=\mathrm{E}\left\{\mathbf{y}_{k}^{m} x_{k}^{*}\right\}=\mathrm{E}\left\{\left[\begin{array}{c}
\mathbf{r}_{k}^{m} x_{k}^{*} \\
\left(\mathbf{r}_{k}^{m}\right)^{*} x_{k}^{*}
\end{array}\right]\right\}=\left[\begin{array}{c}
\mathbf{s}_{k} \\
\mathbf{0}
\end{array}\right] \text {; } \\
& \tilde{\mathbf{E}}_{k}^{m}=\mathrm{E}\left\{\left[\mathbf{x}_{k}-\mathbf{d}_{k}^{m-1}\right]\left[\mathbf{x}_{k}-\mathbf{d}_{k}^{m-1}\right]^{\mathcal{T}}\right\} \\
& =\operatorname{diag}\left\{\left[\begin{array}{lllllll}
\tilde{E}_{1}^{m} & \ldots & \tilde{E}_{k-1}^{m} & 0 & \tilde{E}_{k+1}^{m} & \ldots & \tilde{E}_{N_{t}}^{m}
\end{array}\right]\right\} .
\end{aligned}
$$

The $i$ th diagonal element of $\tilde{\mathbf{E}}_{k}^{m}$ can be calculated as

$$
\begin{aligned}
\tilde{E}_{i}^{m}= & \mathrm{E}\left[\left(x_{i}-d_{i}^{m-1}\right)^{2}\right]=\mathrm{E}\left[x_{i}^{2}\right]-\left(d_{i}^{m-1}\right)^{2} \\
= & \mathrm{E}\left[x_{i, I}^{2}+2 j x_{i, I} x_{i, Q}-x_{i, Q}^{2}\right]-\left(d_{i, I}^{m-1}\right)^{2} \\
& \quad-2 j d_{i, I}^{m-1} d_{i, Q}^{m-1}+\left(d_{i, Q}^{m-1}\right)^{2} \\
= & \mathrm{E}\left[x_{i, I}^{2}\right]-\mathrm{E}\left[x_{i, Q}^{2}\right]+\left(d_{i, Q}^{m-1}\right)^{2}-\left(d_{i, I}^{m-1}\right)^{2},
\end{aligned}
$$




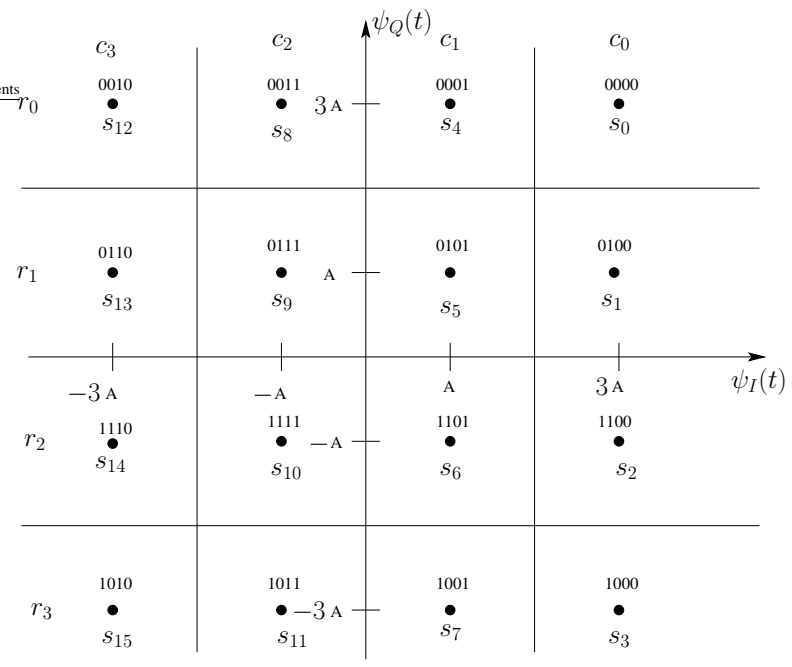

Fig. 1. 16-QAM constellation and symbol-to-bit mapping.

where $\mathrm{E}\left[x_{i, I}^{2}\right]=\sum_{c=0}^{|\mathcal{X}|-1} x_{c, I}^{2} P_{r}\left(x_{i}=s_{c}\right), \mathrm{E}\left[x_{i, Q}^{2}\right]=$ $\sum_{c=0}^{|\mathcal{X}|-1} x_{c, Q}^{2} P_{r}\left(x_{i}=s_{c}\right)$, and $|\mathcal{X}|$ is the modulation level. Note that $\tilde{E}_{i}^{m}=\left(d_{i, Q}^{m-1}\right)^{2}-\left(d_{i, I}^{m-1}\right)^{2}$ in [25], which is valid only for signal constellations with equal energy for in-phase and quadrature components, i.e., $\mathrm{E}\left[x_{i, I}^{2}\right]=$ $\mathrm{E}\left[x_{k, Q}^{2}\right]$. The QPSK system is a typical example for this condition to hold.

Let us express the filter output as $f_{k}^{m}=\left(\boldsymbol{\alpha}_{k}^{m}\right)^{\mathcal{H}} \mathbf{y}_{k}^{m}=$ $\mu_{k}^{m} x_{k}+\nu_{k}^{m} x_{k}^{*}+\eta_{k}^{m}$, where the combined noise and residual interference $\eta_{k}$ can be approximated as a Gaussian random variable [15], [16], i.e., $\eta_{k}^{m} \sim \mathcal{C N}\left(0, N_{\eta}\right)$. In [25], the rotationally variant nature of the random process $\eta_{k}$ has been considered but not utilized since $\mathrm{E}\left[\eta_{k}^{2}\right]=0$ for a sufficiently large spreading factor. Assuming a rotationally invariant zero-mean Gaussian random variable $\eta_{k}^{m}$, its second order statistic is completely characterized by its variance $N_{\eta}=\mathrm{E}\left[\left|\eta_{k}^{m}\right|^{2}\right]$. The parameters $\mu_{k}^{m}, \nu_{k}^{m}, N_{\eta}$ can be computed as

$$
\begin{aligned}
\mu_{k}^{m} & =\mathrm{E}\left\{f_{k}^{m} x_{k}^{*}\right\}=\left(\boldsymbol{\alpha}_{k}^{m}\right)^{\mathcal{H}} \mathrm{E}\left[\mathbf{y} x_{k}^{*}\right]=\left(\boldsymbol{\alpha}_{k}^{m}\right)^{\mathcal{H}}\left[\begin{array}{c}
\mathbf{s}_{k} \\
\mathbf{0}
\end{array}\right] ; \\
\nu_{k}^{m} & =\mathrm{E}\left\{f_{k}^{m} x_{k}\right\}=\left(\boldsymbol{\alpha}_{k}^{m}\right)^{\mathcal{H}} \mathrm{E}\left[\mathbf{y} x_{k}\right]=\left(\boldsymbol{\alpha}_{k}^{m}\right)^{\mathcal{H}}\left[\begin{array}{c}
\mathbf{0} \\
\mathbf{s}_{k}^{*}
\end{array}\right] ; \\
N_{\eta} & =\mathrm{E}\left[\left|\eta_{k}^{m}\right|^{2}\right]=\mathrm{E}\left[\left|f_{k}^{m}-\mu_{k}^{m} x_{k}-\nu_{k}^{m} x_{k}^{*}\right|^{2}\right] \\
& =\mathrm{E}\left\{\left|f_{k}^{m}\right|^{2}\right\}-\left|\mu_{k}^{m}\right|^{2}-\left|\nu_{k}^{m}\right|^{2} \\
& =\left(\mu_{k}^{m}\right)^{*}-\left|\mu_{k}^{m}\right|^{2}-\left|\nu_{k}^{m}\right|^{2} .
\end{aligned}
$$

The above equation holds since $f_{k}^{m}=\left(\boldsymbol{\alpha}_{k}^{m}\right)^{\mathcal{H}} \mathbf{y}$ and $\boldsymbol{\alpha}_{k}^{m}=\boldsymbol{\Psi}_{\mathbf{y y}}^{-1} \boldsymbol{\Psi}_{\mathbf{y} x}$. Therefore,

$$
\begin{aligned}
\mathrm{E}\left\{\left|f_{k}^{m}\right|^{2}\right\} & =\mathrm{E}\left\{\left(\boldsymbol{\alpha}_{k}^{m}\right)^{\mathcal{H}} \mathbf{y} \mathbf{y}^{\mathcal{H}} \boldsymbol{\alpha}_{k}^{m}\right\}=\left(\boldsymbol{\alpha}_{k}^{m}\right)^{\mathcal{H}} \boldsymbol{\Psi}_{\mathbf{y y}} \boldsymbol{\alpha}_{k}^{m} \\
& =\boldsymbol{\Psi}_{\mathbf{y} x}^{\mathcal{H}} \boldsymbol{\Psi}_{\mathbf{y y}}^{-1} \Psi_{\mathbf{y y}} \boldsymbol{\alpha}_{k}^{m}=\boldsymbol{\Psi}_{\mathbf{y} x}^{\mathcal{H}} \boldsymbol{\alpha}_{k}^{m}=\left(\mu_{k}^{m}\right)^{*},
\end{aligned}
$$

which indicates that $\mu_{k}^{m}$ is a real-valued and positive scalar.

After computing the values of $\mu_{k}^{m}, \nu_{k}^{m}$ and $N_{\eta}$, the conditional probability density function (PDF) of the filter output can be obtained as

$$
f\left(f_{k}^{m} \mid x_{k}=s_{c}\right)=\frac{1}{\pi N_{\eta}} \exp \left(-\frac{\left|f_{k}^{m}-\mu_{k}^{m} s_{c}-\nu_{k}^{m} s_{c}^{*}\right|^{2}}{N_{\eta}}\right) .
$$

For a $|\mathcal{X}|=2^{q}$ QAM system, the LLR values for the bits $\left\{v_{k}^{0}, v_{k}^{1}, \ldots, v_{k}^{q-1}\right\}$ are obtained as

$$
\lambda\left(v_{k}^{i}\right)=\ln \frac{f\left(f_{k}^{m} \mid v_{k}^{i}=0\right)}{f\left(f_{k}^{m} \mid v_{k}^{i}=1\right)}=\ln \frac{\sum_{x_{k} \in \mathcal{X}_{i, 0}} f\left(f_{k}^{m} \mid x_{k}\right)}{\sum_{x_{k} \in \mathcal{X}_{i, 1}} f\left(f_{k}^{m} \mid x_{k}\right)},
$$

where $i=0,1, \ldots, q-1$, and $\mathcal{X}_{i, 0}\left(\mathcal{X}_{i, 1}\right)$ is the set of symbols $\left\{s_{c}\right\}$ whose $i$-th corresponding bit takes the value of 0 (1). In the case of 16-QAM modulation, four bits $\left\{v_{k}^{0}, v_{k}^{1}, v_{k}^{2}, v_{k}^{3}\right\}$ are mapped into one of the 16-QAM symbols $s_{c}$. According to the symbol-to-bit mapping as shown in Fig. 1, the LLR values of $v_{k}^{0}$ can be obtained as $\lambda\left(v_{k}^{0}\right)=\ln \frac{f\left(f_{k}^{m} \mid v_{k}^{0}=0\right)}{f\left(f_{k}^{m} \mid v_{k}^{0}=1\right)}=\ln \frac{\sum_{x_{k} \in\left(r_{0}, r_{1}\right)} f\left(f_{k}^{m} \mid x_{k}\right)}{\sum_{x_{k} \in\left(r_{2}, r_{3}\right)} f\left(f_{k}^{m} \mid x_{k}\right)}$, where $x \in\left(r_{i}\right)$ denotes the symbols that lie in the $i^{\text {th }}$ row of the signal constellation. The LLR values of $v_{k}^{1}, v_{k}^{2}, v_{k}^{3}$ can be derived similarly. Utilizing the fact that one term usually dominates each sum, $\lambda\left(v_{k}^{i}\right)$ can be approximated as

$$
\begin{aligned}
\lambda\left(v_{k}^{i}\right) \approx & \ln \frac{\exp \left(-\frac{\left|f_{k}^{m}-\mu_{k}^{m} x_{+}-\nu_{k}^{m} x_{+}^{*}\right|^{2}}{N_{\eta}}\right)}{\exp \left(-\frac{\left|f_{k}^{m}-\mu_{k}^{m} x_{-}-\nu_{k}^{m} x_{-}^{*}\right|^{2}}{N_{\eta}}\right)} \\
= & \frac{1}{N_{\eta}}\left\{\left|f_{k}^{m}-\mu_{k}^{m} x_{-}-\nu_{k}^{m} x_{-}^{*}\right|^{2}\right. \\
& \left.-\left|f_{k}^{m}-\mu_{k}^{m} x_{+}-\nu_{k}^{m} x_{+}^{*}\right|^{2}\right\},
\end{aligned}
$$

where $x_{+}$denotes the symbol corresponding to $\max \left\{f\left(f_{k}^{m} \mid x_{k} \in \mathcal{X}_{i, 0}\right)\right\}$, and $x_{-}$denotes the symbol corresponding to $\max \left\{f\left(f_{k}^{m} \mid x_{k} \in \mathcal{X}_{i, 1}\right)\right\}$.

Assuming independence between the transmitted bits [27] (this is obviously true for uncoded systems, whereas it is also the case for coded systems with interleaving), we have

$$
P_{r}\left(x_{i}\right)=\Pi_{p=0, \ldots, q-1} P_{r}\left(v_{i}^{p}\right) .
$$

where

$$
\begin{aligned}
& P_{r}\left(v_{i}^{p}=0\right)=\frac{e^{\lambda\left(v_{i}^{p}\right)}}{1+e^{\lambda\left(v_{i}^{p}\right)}} \\
& P_{r}\left(v_{i}^{p}=1\right)=\frac{1}{1+e^{\lambda\left(v_{i}^{p}\right)}} .
\end{aligned}
$$

With the a priori probability of each symbol $P_{r}\left(x_{i}\right)$, the soft estimate $d_{i}^{m-1}$ in (3) and the variance $\operatorname{var}\left(x_{i}\right)$ in (4), respectively, can be calculated as [27]

$$
\begin{aligned}
& d_{i}^{m-1}=\mathrm{E}\left\{x_{i}\right\}=\sum_{c=0}^{|\mathcal{X}|-1} s_{c} P_{r}\left(x_{i}=s_{c}\right) \\
& \operatorname{var}\left(x_{i}\right)=\mathrm{E}\left[\left|x_{i}\right|^{2}\right]-\left|\mathrm{E}\left\{x_{i}\right\}\right|^{2},
\end{aligned}
$$

where $\mathrm{E}\left[\left|x_{i}\right|^{2}\right]=\sum_{c=0}^{|\mathcal{X}|-1}\left|s_{c}\right|^{2} P_{r}\left(x_{i}=s_{c}\right)$. 


\section{The proposed ISDIC scheme}

In Section III-B, the interference plus noise term $\eta_{k}$ is regarded as a rotationally invariant random process. However, as will become evident later on, this treatment is sub-optimum for the MIMO systems. Here, we propose an improved scheme which takes into account the rotationally variant nature of $\eta_{k}^{m}$, and utilizes the fact that $\tilde{N}_{\eta}=\mathrm{E}\left[\left(\eta_{k}^{m}\right)^{2}\right] \neq 0$, which can be computed as

$$
\begin{aligned}
\tilde{N}_{\eta} & =\mathrm{E}\left[\left(\eta_{k}^{m}\right)^{2}\right]=\mathrm{E}\left[\left(f_{k}^{m}-\mu_{k}^{m} x_{k}-\nu_{k}^{m} x_{k}^{*}\right)^{2}\right] \\
& =\mathrm{E}\left[\left(f_{k}^{m}-\mu_{k}^{m} x_{k}-\nu_{k}^{m} x_{k}^{*}\right)\left(f_{k}^{m}-\mu_{k}^{m} x_{k}-\nu_{k}^{m} x_{k}^{*}\right)\right] \\
& =\mathrm{E}\left\{\left(f_{k}^{m}\right)^{2}\right\}-2 \mu_{k}^{m} \nu_{k}^{m} \\
& =\mathrm{E}\left\{\left(\boldsymbol{\alpha}_{k}^{m}\right)^{\mathcal{H}} \mathbf{y}_{k}^{m}\left(\mathbf{y}_{k}^{m}\right)^{\mathcal{T}}\left(\boldsymbol{\alpha}_{k}^{m}\right)^{*}\right\}-2 \mu_{k}^{m} \nu_{k}^{m} \\
& =\left(\boldsymbol{\alpha}_{k}^{m}\right)^{\mathcal{H}} \tilde{\boldsymbol{\Psi}}_{\mathbf{y y}}\left(\boldsymbol{\alpha}_{k}^{m}\right)^{*}-2 \mu_{k}^{m} \nu_{k}^{m} .
\end{aligned}
$$

The above equation holds since $\left(\boldsymbol{\alpha}_{k}^{m}\right)^{\mathcal{H}} \mathbf{y}_{k}^{m}=$ $\left(\mathbf{y}_{k}^{m}\right)^{\mathcal{T}}\left(\boldsymbol{\alpha}_{k}^{m}\right)^{*}$. Therefore

$$
\begin{aligned}
\mathrm{E}\left\{\left(f_{k}^{m}\right)^{2}\right\} & =\mathrm{E}\left\{\left(\boldsymbol{\alpha}_{k}^{m}\right)^{\mathcal{H}} \mathbf{y}_{k}^{m}\left(\mathbf{y}_{k}^{m}\right)^{\mathcal{T}}\left(\boldsymbol{\alpha}_{k}^{m}\right)^{*}\right\} \\
& =\left(\boldsymbol{\alpha}_{k}^{m}\right)^{\mathcal{H}} \mathrm{E}\left\{\mathbf{y}_{k}^{m}\left(\mathbf{y}_{k}^{m}\right)^{\mathcal{T}}\right\}\left(\boldsymbol{\alpha}_{k}^{m}\right)^{*} \\
& =\left(\boldsymbol{\alpha}_{k}^{m}\right)^{\mathcal{H}} \tilde{\boldsymbol{\Psi}}_{\mathbf{y y}}\left(\boldsymbol{\alpha}_{k}^{m}\right)^{*},
\end{aligned}
$$

where

$$
\begin{aligned}
\tilde{\mathbf{\Psi}}_{\mathbf{y y}} & =\mathrm{E}\left\{\mathbf{y}_{k}^{m}\left(\mathbf{y}_{k}^{m}\right)^{\mathcal{T}}\right\}=\mathrm{E}\left\{\left[\begin{array}{c}
\mathbf{r}_{k}^{m} \\
\left(\mathbf{r}_{k}^{m}\right)^{*}
\end{array}\right]\left[\left(\mathbf{r}_{k}^{m}\right)^{\mathcal{T}} \quad\left(\mathbf{r}_{k}^{m}\right)^{\mathcal{H}}\right]\right\} \\
& =\left[\begin{array}{cc}
\mathbf{S} \tilde{\mathbf{E}}_{k}^{m} \mathbf{S}^{\mathcal{T}} & \mathbf{S E}_{k}^{m} \mathbf{S}^{\mathcal{H}}+\sigma_{n}^{2} \mathbf{I} \\
\mathbf{S}^{*} \mathbf{E}_{k}^{m} \mathbf{S}^{\mathcal{T}}+\sigma_{n}^{2} \mathbf{I} & \mathbf{S}^{*}\left(\tilde{\mathbf{E}}_{k}^{m}\right)^{*} \mathbf{S}^{\mathcal{H}}
\end{array}\right]
\end{aligned}
$$

Denoting $f_{k}^{m}=f_{k, I}^{m}+j f_{k, Q}^{m}, x_{k}=x_{k, I}+j x_{k, Q}$, $\nu_{k}^{m}=\nu_{k, I}^{m}+j \nu_{k, Q}^{m}$, and $\eta_{k}^{m}=\eta_{k, I}^{m}+j \eta_{k, Q}^{m}$, the filter output $f_{k}^{m}=\mu_{k}^{m} x_{k}+\nu_{k}^{m} x_{k}^{*}+\eta_{k}^{m}$ can be reformed as

$$
\underbrace{\left[\begin{array}{c}
f_{k, I}^{m} \\
f_{k, Q}^{m}
\end{array}\right]}_{\mathbf{f}_{k}^{m}}=\underbrace{\left[\begin{array}{c}
\left(\mu_{k}^{m}+\nu_{k, I}^{m}\right) x_{k, I}+\nu_{k, Q}^{m} x_{k, Q} \\
\left(\mu_{k}^{m}-\nu_{k, I}^{m}\right) x_{k, Q}+\nu_{k, Q}^{m} x_{k, I}
\end{array}\right]}_{\mathbf{x}_{k}}+\underbrace{\left[\begin{array}{c}
\eta_{k, I}^{m} \\
\eta_{k, Q}^{m}
\end{array}\right]}_{\boldsymbol{\eta}_{k}^{m}} .
$$

Since the probability distribution of a complex random variable or vector is a joint distribution of its real and imaginary parts, we have

$$
\begin{aligned}
& f\left(f_{k}^{m} \mid x_{k}\right)=f\left(\mathbf{f}_{k}^{m} \mid \mathbf{x}_{k}\right) \\
& =\frac{1}{2 \pi \sqrt{\operatorname{det} \boldsymbol{\Omega}_{k}^{m}}} \exp \left(-\frac{1}{2}\left(\mathbf{f}_{k}^{m}-\mathbf{x}_{k}\right)^{\mathcal{H}}\left(\boldsymbol{\Omega}_{k}^{m}\right)^{-1}\left(\mathbf{f}_{k}^{m}-\mathbf{x}_{k}\right)\right),
\end{aligned}
$$

where the covariance matrix of the Gaussian noise is $\boldsymbol{\Omega}_{k}^{m}=\mathrm{E}\left[\boldsymbol{\eta}_{k}^{m}\left(\boldsymbol{\eta}_{k}^{m}\right)^{\mathcal{H}}\right]$. Define a mapping matrix as $\mathbf{J}=$ $\frac{1}{\sqrt{2}}\left[\begin{array}{cc}1 & j \\ 1 & -j\end{array}\right]$, which is an unitary matrix since $\mathbf{J} \mathbf{J}^{\mathcal{H}}=$ $\mathbf{J}^{\mathcal{H}} \mathbf{J}=\mathbf{I}$, and $\mathbf{J}^{-1}=\mathbf{J}^{\mathcal{H}}$. Therefore,

$$
\begin{aligned}
\mathbf{J} \boldsymbol{\Omega}_{k}^{m} \mathbf{J}^{\mathcal{H}} & =\mathbf{J} \mathrm{E}\left[\boldsymbol{\eta}_{k}^{m}\left(\boldsymbol{\eta}_{k}^{m}\right)^{\mathcal{H}}\right] \mathbf{J}^{\mathcal{H}}=\mathrm{E}\left[\left(\mathbf{J} \boldsymbol{\eta}_{k}^{m}\right)\left(\mathbf{J} \boldsymbol{\eta}_{k}^{m}\right)^{\mathcal{H}}\right] \\
& =\frac{1}{2} \mathrm{E}\left[\gamma_{k}^{m}\left(\gamma_{k}^{m}\right)^{\mathcal{H}}\right]=\frac{1}{2} \boldsymbol{\Gamma}_{k}^{m},
\end{aligned}
$$

where $\gamma_{k}^{m}=\left[\begin{array}{c}\eta_{k}^{m} \\ \left(\eta_{k}^{m}\right)^{*}\end{array}\right]$, and $\boldsymbol{\Gamma}_{k}^{m}=\mathrm{E}\left[\boldsymbol{\gamma}_{k}^{m}\left(\boldsymbol{\gamma}_{k}^{m}\right)^{\mathcal{H}}\right]=$ $\mathrm{E}\left\{\left[\begin{array}{cc}\eta_{k}^{m}\left(\eta_{k}^{m}\right)^{*} & \eta_{k}^{m} \eta_{k}^{m} \\ \left(\eta_{k}^{m}\right)^{*}\left(\eta_{k}^{m}\right)^{*} & \left(\eta_{k}^{m}\right)^{*} \eta_{k}^{m}\end{array}\right]\right\}=\left[\begin{array}{cc}N_{\eta} & \tilde{N}_{\eta} \\ \tilde{N}_{\eta}^{*} & N_{\eta}\end{array}\right]$, where $N_{\eta}$ and $\tilde{N}_{\eta}$ are given by (8) and (10), respectively.
From (14), we have $\boldsymbol{\Omega}_{k}^{m}=\frac{1}{2} \mathbf{J}^{\mathcal{H}} \boldsymbol{\Gamma}_{k}^{m} \mathbf{J}$, therefore, $\left(\boldsymbol{\Omega}_{k}^{m}\right)^{-1}=2 \mathbf{J}^{\mathcal{H}}\left(\boldsymbol{\Gamma}_{k}^{m}\right)^{-1} \mathbf{J}$. The PDF in (13) can thus be reformed as $f\left(f_{k}^{m} \mid x_{k}\right)=\frac{1}{2 \pi \sqrt{\operatorname{det} \boldsymbol{\Omega}_{k}^{m}}} \exp \left[-\left(\mathbf{f}_{k}^{m}-\right.\right.$ $\left.\mathbf{x}_{k}\right)^{\mathcal{H}} \mathbf{J}^{\mathcal{H}}\left(\boldsymbol{\Gamma}_{k}^{m}\right)^{-1} \mathbf{J}\left(\mathbf{f}_{k}^{m}-\mathbf{x}_{k}\right)$ ]. The LLR value of $v_{k}^{i}$ can thus be computed as

$$
\begin{aligned}
\lambda\left(v_{k}^{i}\right)= & \ln \frac{f\left(f_{k}^{m} \mid v_{k}^{i}=0\right)}{f\left(f_{k}^{m} \mid v_{k}^{i}=1\right)} \\
\approx & \ln \frac{\exp \left[-\left(\mathbf{f}_{k}^{m}-\mathbf{x}_{+}\right)^{\mathcal{H}} \mathbf{J}^{\mathcal{H}}\left(\boldsymbol{\Gamma}_{k}^{m}\right)^{-1} \mathbf{J}\left(\mathbf{f}_{k}^{m}-\mathbf{x}_{+}\right)\right]}{\exp \left[-\left(\mathbf{f}_{k}^{m}-\mathbf{x}_{-}\right)^{\mathcal{H}} \mathbf{J}^{\mathcal{H}}\left(\boldsymbol{\Gamma}_{k}^{m}\right)^{-1} \mathbf{J}\left(\mathbf{f}_{k}^{m}-\mathbf{x}_{-}\right)\right]} \\
= & \left(\mathbf{f}_{k}^{m}-\mathbf{x}_{-}\right)^{\mathcal{H}} \mathbf{J}^{\mathcal{H}}\left(\boldsymbol{\Gamma}_{k}^{m}\right)^{-1} \mathbf{J}\left(\mathbf{f}_{k}^{m}-\mathbf{x}_{-}\right) \\
& -\left(\mathbf{f}_{k}^{m}-\mathbf{x}_{+}\right)^{\mathcal{H}} \mathbf{J}^{\mathcal{H}}\left(\boldsymbol{\Gamma}_{k}^{m}\right)^{-1} \mathbf{J}\left(\mathbf{f}_{k}^{m}-\mathbf{x}_{+}\right) .
\end{aligned}
$$

In (15), $\mathbf{x}_{+}$denotes the vector $\mathbf{x}_{k}$ corresponding to $\max \left\{f\left(\mathbf{f}_{k}^{m} \mid \mathbf{x} \in \mathbf{X}_{i, 0}\right)\right\}$ and $\mathbf{x}_{-}$denotes the vector $\mathbf{x}_{k}$ corresponding to $\max \left\{f\left(\mathbf{f}_{k}^{m} \mid \mathbf{x} \in \mathbf{X}_{i, 1}\right)\right\}$, where $\mathbf{X}_{i, 0}$ $\left(\mathbf{X}_{i, 1}\right)$ is derived from $\mathcal{X}_{i, 0}\left(\mathcal{X}_{i, 1}\right)$ according to the vector formation in Eq. (12).

The above procedure can also be incorporated into the V-BLAST MMSE decision feedback detection (VMMSEDFD) scheme introduced in [28], [29]. Let the ordered set $O \equiv\left\{k_{1}, k_{2}, \ldots, k_{N_{t}}\right\}$ be a permutation of the integer $1,2, \ldots, N_{t}$ specifying the order in which the transmitted symbols are detected, and $\mathbf{s}_{j}$ denote the $j$ th column of $\mathbf{S}$. To detect the $k_{i}$ th symbol with the improved VMMSEDFD algorithm, $\mathbf{S E}_{k}^{m} \mathbf{S}^{\mathcal{H}}+\sigma_{n}^{2} \mathbf{I}$ and $\mathbf{S} \tilde{E}_{k}^{m} \mathbf{S}^{\mathcal{T}}$ in (6) and (11) should be replaced by $\sum_{j=k_{1}}^{k_{i-1}} \mathbf{s}_{j} \mathbf{s}_{j}^{\mathcal{H}} \operatorname{var}\left(x_{k_{j}}\right)+$ $\sum_{j=k_{i}}^{k_{N_{t}}} \mathbf{s}_{j} \mathbf{s}_{j}^{\mathcal{H}}+\sigma_{n}^{2} \mathbf{I}$ and $\sum_{j=k_{1}}^{k_{i-1}} \mathbf{s}_{j} \mathbf{s}_{j}^{\mathcal{T}}\left(\mathrm{E}\left[x_{k_{j}, I}^{2}\right]-\mathrm{E}\left[x_{k_{j}, Q}^{2}\right]+\right.$ $\left.\left(\bar{d}_{k_{j}, Q}\right)^{2}-\left(\bar{d}_{k_{j}, I}\right)^{2}\right)$, respectively.

The complexity of different algorithms is compared in Table I, which shows the required number of complex multiplications/divisions, and additions/subtractions for one symbol estimate corresponding to the calculation of LLRs for $q=\log _{2}|\mathcal{X}|$ bits, where $|\mathcal{X}|$ is the modulation level. Here we assume the number of antennas $N_{t}=N_{r}=N$. It can be seen from the table that the complexity of the proposed scheme is comparable to that of the modified ISDIC, both having higher complexity than the conventional scheme. However, the difference is not significant, all the schemes have a complexity which is cubic in the number of transmit antennas. As will be shown in the next section, the complexity increase by the proposed scheme is largely compensated by the significant performance improvements.

\section{NUMERICAL RESULTS}

In this section, we mainly compare three different iterative receivers: i) the conventional iterative MIMO receiver, i.e., ISDIC with the linear MMSE filter [14]-[16] introduced in Section III-A; ii) the ISDIC with the modified MMSE filter [25] introduced in Section III-B; iii) the proposed ISDIC receiver introduced in Section III-C. An uncoded MIMO system with 4 transmit and $3-5$ receive antennas is employed in our simulations. We assume an uncorrelated Rayleigh fading channel between each pair of transmit and receive antennas, and the receiver has perfect knowledge of the channel state information (CSI). 
TABLE I

COMPLEXITY FOR ONE SYMBOL ESTIMATE AT ONE ITERATION FOR THE ALGORITHMS CONSIDERED.

\begin{tabular}{|c|c|c|}
\hline \hline operations & $\times / \div$ & $+/-$ \\
\hline C-ISDIC & $3 N^{3}+6 N^{2}+2 N+2|\mathcal{X}|$ & $3 N^{3}+2 N^{2}+N+|\mathcal{X}|+\log _{2}|\mathcal{X}|-2$ \\
\hline M-ISDIC & $18 N^{3}+16 N^{2}+6 N+4|\mathcal{X}|+6$ & $18 N^{3}+4 N^{2}+4 N+|\mathcal{X}|+\log _{2}|\mathcal{X}|-5$ \\
\hline Proposed & $18 N^{3}+16 N^{2}+8 N+2|\mathcal{X}|^{2}+6|\mathcal{X}|+28$ & $18 N^{3}+4 N^{2}+6 N+|\mathcal{X}|^{2}+2|\mathcal{X}|+\log _{2}|\mathcal{X}|+13$ \\
\hline
\end{tabular}

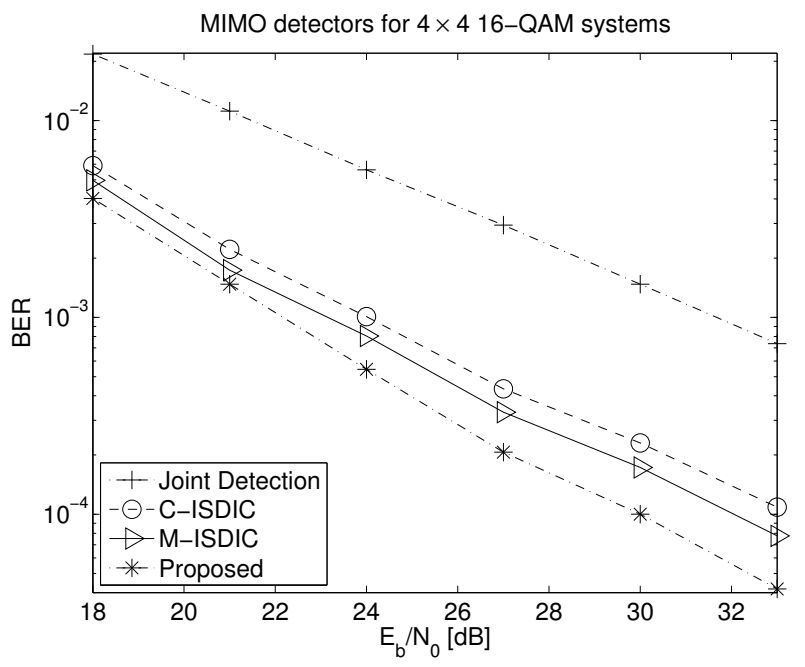

Fig. 2. Performance comparison of different MIMO detectors for 16QAM systems. The curves (excluding the one corresponding to the joint detection) represent the performance of different iterative schemes at the 3rd iteration when the systems reach the convergence.

The channel coefficients are normalized such that the average channel gain for each transmitted symbol is equal to unity. To study the behavior of each algorithm, the number of iterations is set to 3 since it is observed that all the algorithms would converge after 3 iterations.

Fig. 2 shows the performance comparison for the 16QAM system with a $4 \times 4$ antenna setup. The modified ISDIC (M-ISDIC) scheme outperforms the conventional ISDIC (C-ISDIC) scheme by taking into account the rotationally variant residual interference as a result of the interference cancellation process. However, both are outperformed by the proposed scheme which further exploits the rotational variance property at the filter output. At target $\mathrm{BER}=10^{-4}$, it outperforms the C-ISDIC and M-ISDIC scheme by approximately 3 and $2 \mathrm{~dB}$, respectively. The gain is smaller at low SNRs due to the dominance of the rotationally invariant channel noise. As SNR increases, the performance gain by the proposed detector becomes larger since it benefits more from exploiting the rotational variance of the interference. The curve for 'Joint Detection' is obtained by linear MMSE detector [29] which jointly detects all the symbols from different transmit antennas. It is evident from the figure that this non-iterative scheme has much worse performance than the other iterative schemes, which indicates the effectiveness of iterative detection in mitigating the co-channel interference in MIMO systems.

The convergence behavior of the proposed MIMO

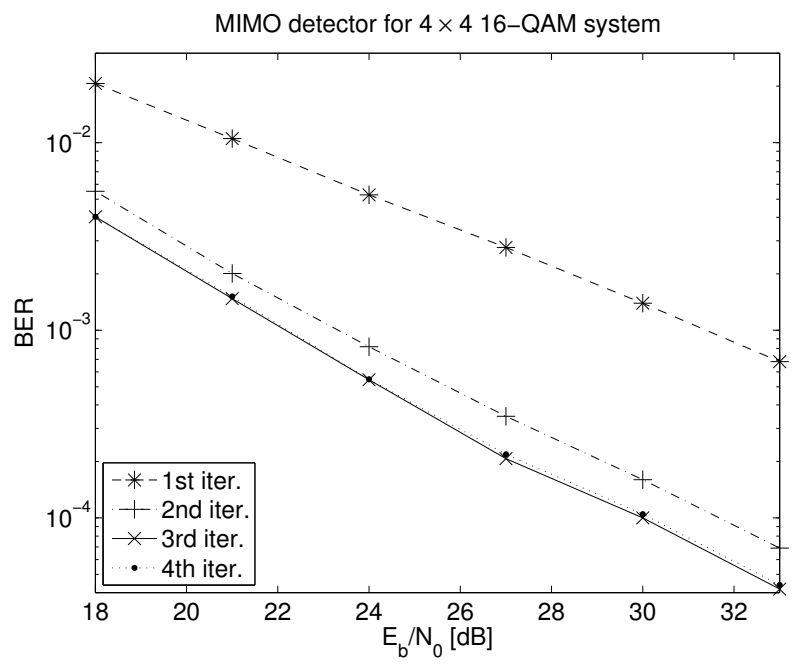

Fig. 3. Convergency behavior of the proposed MIMO detector.

detector is studied in Fig. 3. One can see from the figure that it takes 3 iterations for the algorithm to reach steady state, further iterations do not yield noticeable performance improvement. The most significant gain is obtained at the second iteration.

In Fig. 4, we compare the performance of the conventional and the proposed ISDIC schemes with their VMMSE-FDE counterparts in $4 \times 416-\mathrm{QAM}$ systems. The conventional VMMSE-FDE is implemented according to [28], [29], and the proposed VMMSE-FDE is implmented according to the description at the end of Section III-C. The nulling vectors are designed under the MMSE criterion with the optimal detection order. One can see from the figure that VMMSE-FDE is slightly better than ISDIC with the conventional design, however, it is outperformed by ISDIC with the proposed method assuming rotationally variant interference plus noise component. The gap can be up to $1 \mathrm{~dB}$ at low BER, meaning that that parallel interference cancellation with the proposed design can better mitigate the error propagation problem than successive interference cancellation employed by VMMSE-FDE.

In Fig. 5, we compare different MIMO detection schemes for QPSK systems with $4 \times 3,4 \times 4$ and $4 \times 5$ MIMO configurations. Simulations results demonstrate noticeable performance improvements using our proposed receiver in all the cases. The performance gain is most notable with the $4 \times 3$ configuration. The proposed scheme enables the system to operate reliably in such an underdetermined system, which is not possible with the conventional receiver. However, the gain decreases as the 


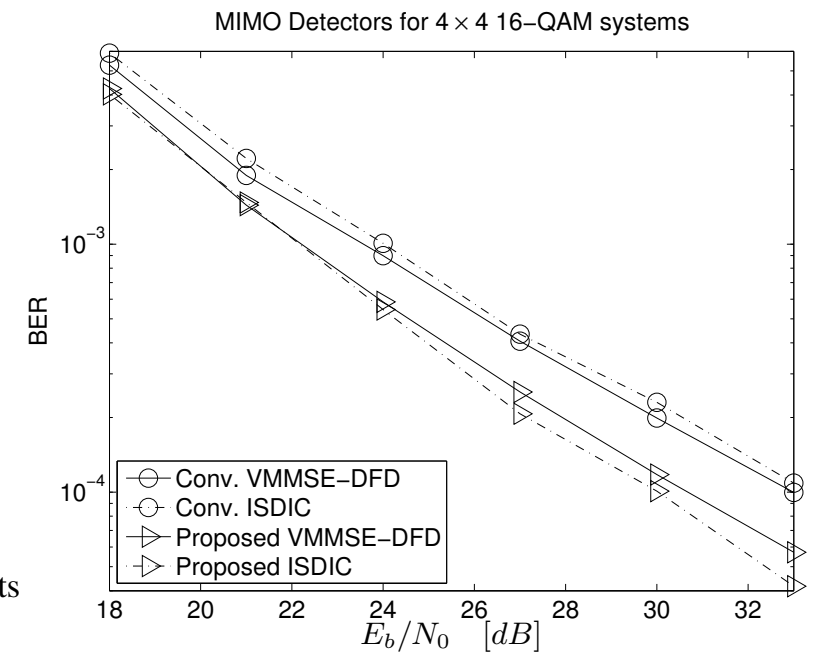

Fig. 4. Performance comparison of between ISDIC and VMMSE-DFD for 16-QAM systems. The ISDIC curves represent the performance at the 3rd iteration.

number of receive antenna increases, e.g., a performance gain of $3 \mathrm{~dB}$ can be observed by the proposed ISDIC compared to the conventional ISDIC at target $\mathrm{BER}=10^{-4}$ in the $4 \times 4$ MIMO system; whereas only $0.9 \mathrm{~dB}$ gain can be achieved in the $4 \times 5$ MIMO system. Apparently, it is more advantageous to apply the proposed iterative detection scheme to underdetermined MIMO systems where there are more transmit antennas than receive antennas.

In Fig. 6, we extend the proposed detection scheme to coded systems and compare its performance with that of the conventional Turbo-MIMO scheme [14]. In our experiments, we use per-antenna-coding, i.e., the incoming bit stream is first transformed to $N_{t}=4$ parallel substreams (each substream length is 2720 bits), and channel encoding, interleaving and modulation are performed separately for each substream. The employed convolutional code has a constraint length of 5 and generator polynomial $(23,35)_{8}$. The modulation scheme is QPSK. Figs. 6(a) and 6(b) show the performance comparison for $4 \times 2$ and $4 \times 3$ MIMO systems, respectively. A performance gain of $1.2 \mathrm{~dB}$ is observed by applying the proposed iterative detection scheme compared to the conventional Turbo-MIMO in $4 \times 2$ MIMO systems at target $\mathrm{BER}=10^{-4}$. However, the gap is smaller in $4 \times 3$ MIMO systems, e.g., the gain is about $0.9 \mathrm{~dB}$ at target $\mathrm{BER}=10^{-6}$. No noticeable gains have been observed for $4 \times 4$ MIMO systems. The results obtained for the coded MIMO systems also indicate the advantage of applying the proposed scheme to underdetermined MIMO systems.

\section{CONCLUSIONS}

We have proposed a novel MIMO detector with enhanced interference and noise estimation. By exploiting the rotationally variant property of the interference canceled signal at both the input and output of the MMSE filter, the proposed scheme achieves superior performance than its conventional counterparts. A performance gain up to $3 \mathrm{~dB}$ at target bit error rate of $10^{-4}$ has been observed for the 16-QAM and QPSK modulated $4 \times 4$ MIMO systems compared to the conventional iterative MIMO detector. We also observed that it is more advantageous to apply the proposed scheme to an underdetermined MIMO system than an overdetermined system, and the performance gain is more notable in uncoded MIMO systems than in coded MIMO systems.

\section{ACKNOWLEDGEMENT}

We wish to thank the anonymous reviewers for providing us valuable and constructive comments and helping us improve the quality of this paper.

$$
\begin{aligned}
\epsilon & =\mathrm{E}\left[\left\|\boldsymbol{\zeta} \mathbf{r}+\boldsymbol{\eta} \mathbf{r}^{*}-\mathbf{s}\right\|^{2}\right]=\mathrm{E}\left[\left\|\boldsymbol{\alpha}^{\mathcal{H}} \mathbf{y}-\mathbf{s}\right\|^{2}\right] \\
& =\boldsymbol{\alpha}^{\mathcal{H}} \mathbf{R}_{\mathbf{y}} \boldsymbol{\alpha}-\boldsymbol{\alpha}^{\mathcal{H}} \mathbf{T}_{\mathbf{y}}-\mathbf{T}_{\mathbf{y}}^{\mathcal{H}} \boldsymbol{\alpha}+\mathbf{I}_{N},
\end{aligned}
$$

where $\boldsymbol{\zeta}, \boldsymbol{\eta}$ are $1 \times K N$ row vectors, $\boldsymbol{\alpha}=\left[\begin{array}{ll}\boldsymbol{\zeta} & \boldsymbol{\eta}\end{array}\right]^{\mathcal{H}}$, $\mathbf{y}=\left[\begin{array}{ll}\mathbf{r}^{\mathcal{T}} & \mathbf{r}^{\mathcal{H}}\end{array}\right]^{\mathcal{T}}$,

\section{REFERENCES}

[1] E. Telatar. "Capacity of multi-antenna Gaussian channels", European Transactions on Telecommunications, vol. 10, no. 6, pp. 585-595, Nov. 1999

[2] G. Foschini. "Layered space-time architecture for wireless communication in a fading environment when using multiple-element antennas", Bells Labs Tech. J., vol. 1, pp. 41-59, 1996.

[3] P. Wolniansky, G. Foschini, G. Golden, R. Valenzuela. "V-BLAST: an architecture for realizing very high data rates over the richscattering wireless channel", IEEE ISSSE, pp. 295-300, Sept. 1998.

[4] L. Barbero, J. Thompson. "Fixing the complexity of the sphere decoder for MIMO detection", IEEE Transactions on Wireless Communications, vol. 7, no. 6, pp. 2131-2141, June 2008.

[5] D. Milliner, E. Zimmermann, J. Barry, G. Fettweis. "A fixedcomplexity smart candidate adding algorithm for soft-out MIMO detection", IEEE Journal of Selected Topics in Signal Processing, vol. 3 no. 6, pp. 1016-1025, Dec. 2009.

[6] Y. Li, J. Moon. "Reduced-complexity soft MIMO detection based on causal and noncausal detection feedback", IEEE Transactions on Signal Processing, vol. 56, no. 3, pp. 1178-1187, March 2008.

[7] T. Liu, Y. Liu. "Modified fast recursive algorithm for efficient MMSE-SIC detection of the V-BLAST system", IEEE Transactions on Wireless Communications, vol. 7, no. 10, pp. 3713-3717, Oct. 2008.

[8] T. Liu. "Some results for the fast MMSE-SIC detection in spatially multiplexed MIMO systems", IEEE Transactions on Wireless Communications, vol. 8, no. 11, pp. 5443-5448, Nov. 2009.

[9] A. Alimohmmad, S. Fard, B. Cockburn. "Improved layered MIMO detection algorithm with near-optimal performance", IET Electronic Letters, vol. 45, no. 13, pp. 675-676, June 2009.

[10] J. Choi, H. Nguyen. "SIC-based detection with list and lattice redution for MIMO channels", IEEE Transactions on Vehicular Technology, vol. 58, no. 7, pp. 3786-3790, Sept. 2009.

[11] J. Choi, A. Singer, J. Lee, N. Cho. "Improved linear soft-input soft-output detection via soft feedback successive interference cancellation", IEEE Transactions on Communications, vol. 58, no. 3, pp. 986-996, March 2010.

[12] J. Hagenauer. "The turbo principle: tutorial introduction and state of the art". Proc. International Symposium on Turbo Codes, pp. 1-11, Sept. 1997.

[13] G. Foschini, M. Gans. "On limits of wireless communications in a fading environment when using multiple antennas", Wireless Personal Commun., vol. 6, no. 3, pp. 311-335, March 1998.

[14] M. Sellathurai, S. Haykin. "Turbo-BLAST for wireless communications: theory and experiments", IEEE Trans. Sig. Proc., vo. 50, no. 10, pp. 2538-2546, Oct. 2002. 


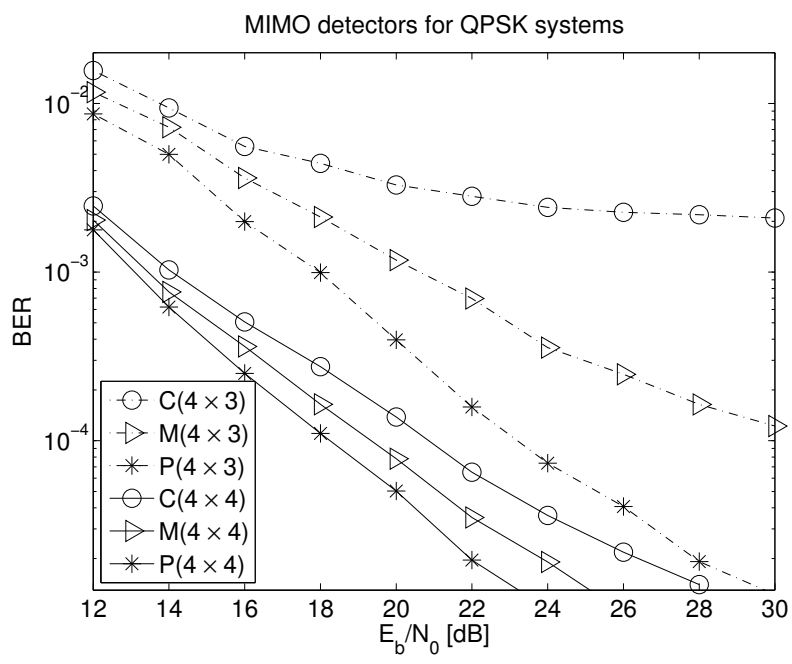

(a) $4 \times 3$ and $4 \times 4$ systems.

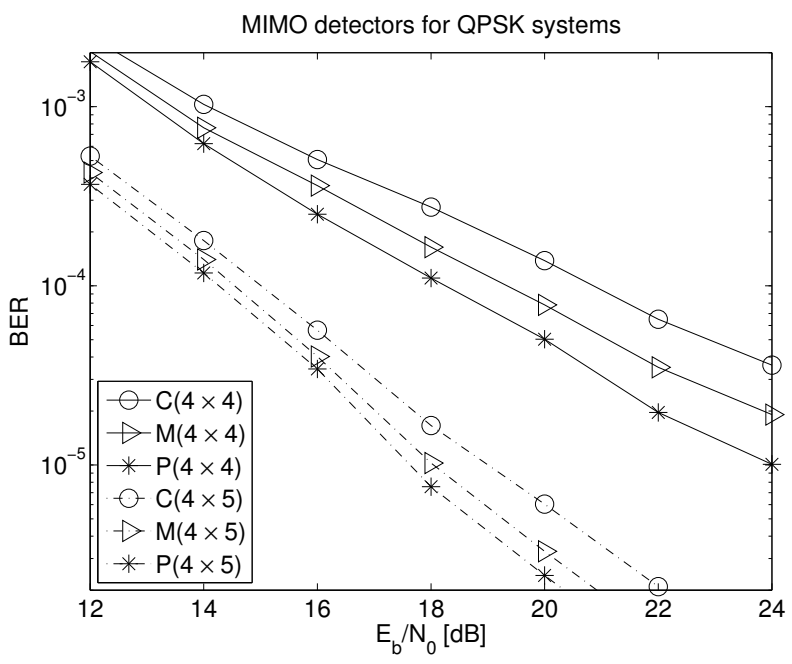

(b) $4 \times 4$ and $4 \times 5$ systems

Fig. 5. Performance comparison of MIMO detectors for QPSK systems with different antenna configurations. The curves represent the performance of different detection schemes at the 3rd iteration. Notations: 'C' - Conventional ISDIC; 'M' - Modified ISDIC; 'P': Proposed ISDIC.

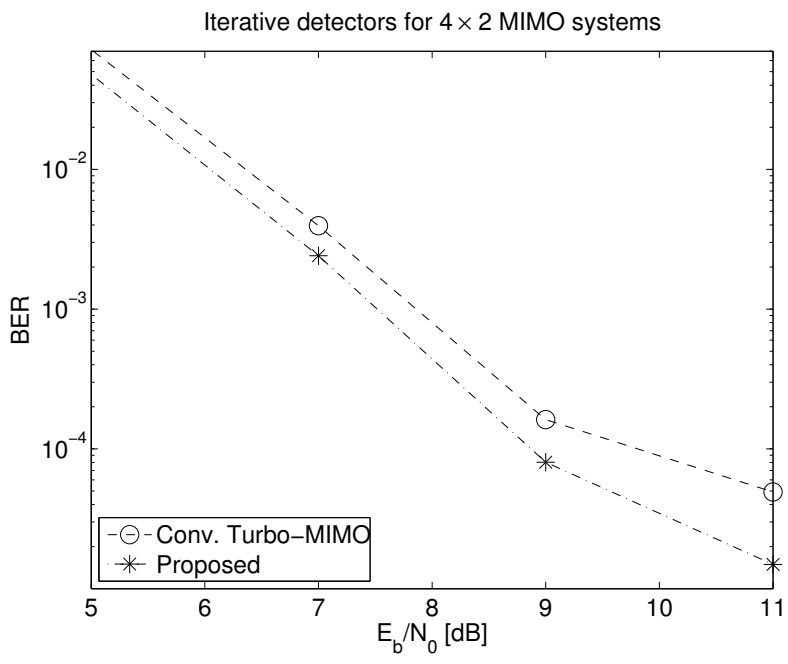

(a) $4 \times 2$ MIMO systems.

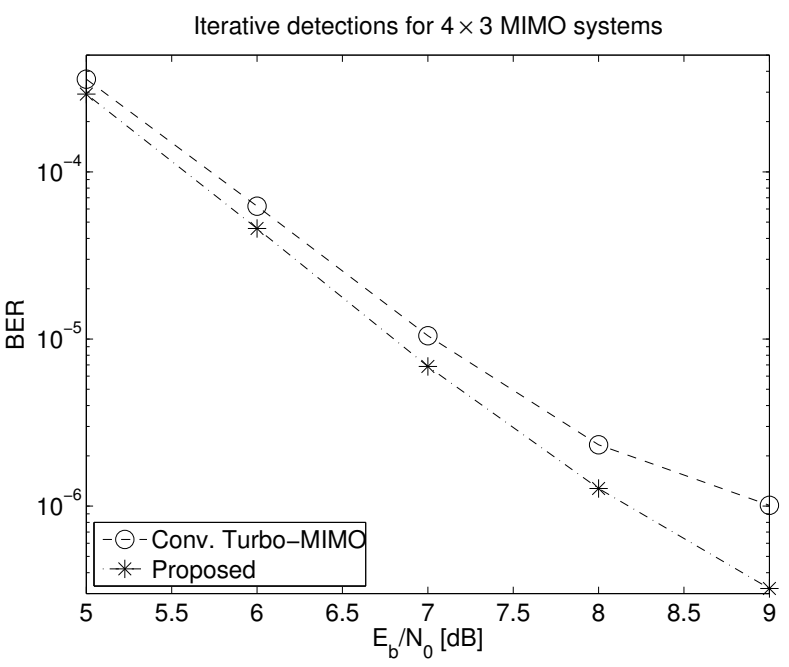

(b) $4 \times 3$ MIMO systems

Fig. 6. Performance comparison between the conventional Turbo-MIMO scheme and the proposed Turbo-MIMO scheme. The curves for the $4 \times 2$ MIMO systems represent the performance of different schemes at the 5 th iteration, the curves for the $4 \times 3 \mathrm{MIMO}$ systems represent the performance of different schemes at the 4th iteration when the systems reach convergence.

[15] X. Wautelet, A. Dejonghe, L. Vandendorpe. "MMSE-based fractional turbo receiver for space-time BICM over frequencyselective MIMO fading channels", IEEE Trans. Sig. Proc., vo. 52, no. 6, pp. 1804-1809, June 2004.

[16] J. Wang, S. Li. "Reliability based reduced-complexity MMSE soft interference cancellation MIMO turbo receiver", Proc. PIMRC, pp. 1-4, Sept. 2007.

[17] H. Lee, B. Lee, I. Lee. "Iterative detection and decoding with an improved V-BLAST for MIMO-OFDM", IEEE Journal on Selected Areas in Communications, vol. 24, no. 3, pp. 504-513, March 2006.

[18] S. Ahmed, T. Ratnarajah, M. Sellathurai, C. Cowan. "Reducedcomplexity iterative equalization for severe time-dispersive MIMO channels", IEEE Transactions on Vehicular Technology, vol. 57, no. 1, pp. 594-600, Jan. 2008.

[19] A. Nakajima, D. Garg, F. Adachi. "Turbo coded MIMO multiplexing with iterative soft parallel interference cancellation", Proc. IEEE Vehicular Technology Conference, pp. 1410-1414, Sept. 2004.

[20] B. Picinbono, P. Chevalier. "Widely linear estimation with complex data", IEEE Transactions on Signal Processing, vol. 43, no. 8, pp. 2030-2033, August 1995.

[21] D. Mattera, L. Paura, F. Sterle. "Widely linear MMSE equaliser for MIMO linear time-dispersive channel”, Electronic Letters, vol. 39, no. 20, pp. 1481-1482, Oct. 2003.

[22] D. Mattera, L. Paura, F. Sterle. "Widely linear decision-feedback equalizer for time-dispersive linear MIMO channel", IEEE Transactions on Signal Processing, vol. 53, no. 7, pp. 2525-2536, July 
2005.

[23] W. Gerstacker, F. Obernosterer, R. Schober, A. Lehmann, A. Lampe, P. Gunreben. "Equalization concepts for Alamouti's spacetime block code", IEEE Transactions on Communications, vol. 52, no. 7, pp. 1178-1190, July 2004.

[24] M. Witzke, S. Baro, J. Hagenauer. "Iterative detection of generalized coded MIMO signals using a widely linear detector", IEEE Global Telecommunications Conference, vol. 4, pp. 1821-1825, Dec. 2003.

[25] A. Lampe, R. Scholber, W. Gerstacker, J. Huber. "A novel iterative multiuser detector for complex modulation schemes", IEEE Journal on Selected Areas in Communications, vol. 20, no. 2, pp. 339-350, Feb. 2002.

[26] S. Kay. Fundamentals of statistical signal processing, Prentice Hall, NJ, 1998.

[27] A. Dejonghe and L. Vandendorpe. "Turbo-equalization for multilevel modulation: an efficient low-complexity scheme", IEEE International Conference on Communications, vol. 3, pp. 1863 1867, 2002.

[28] G. Golden, G. Foschini, R. Valenzuela, P. Wolniansky. "Detection algorithm and initial laboratory results using the V-BLAST spacetime communication architecture", IEE Electronic Letters, vol. 35, no. 1, pp. 14-16, January 1999.

[29] C. Swetman, J. Thompson, B. Mulgrew, P. Grant. "A comparison of the MMSE detector and its BLAST versions for MIMO channels", IEE Seminar on MIMO: Communications Systems from Concept to Implementations, pp. 19/1 - 19/6, Dec. 2001. 